\title{
Case Report: A Mitochondrial Disorder in a Middle Age Ira- nian Patient: Report of a Rare Case
}

\author{
Mostafa Almasi ${ }^{1 *}$, Mohammad Reza Motamed ${ }^{1}$, Masoud Mehrpour ${ }^{1}$, Bahram Haghi-Ashtiani ${ }^{1}$, Fahimeh Haji Akhondi ${ }^{1}$, Yalda Nilipour ${ }^{2}$ \\ Seyed-Mohammad Fereshtehnejad ${ }^{3}$
}

1. Department of Neurology, Firoozgar Hospital, Iran University of Medical Sciences, Tehran, Iran.

2. Pediatric Pathology Research Center, Mofid Children Hospital, Shahid Beheshti University of Medical Sciences, Tehran, Iran

3. Department of Neurobiology, Care Sciences and Society, Karolinska Institutet, Stockholm, Sweden.

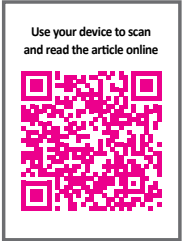

chttation: Almasi, M., Motamed, M. R., Mehrpour, M., Haghi-Ashtiani, B., Haji Akhondi, F., Nilipour, Y., et al. (2017). A Mitochondrial Disorder in a Middle Age Iranian Patient: Report of a Rare Case. Basic and Clinical Neuroscience, 8(4), 337341. https://doi.org/10.18869/nirp.bcn.8.4.337

https://doi.org/10.18869/nirp.ben.8.4.337

Article info:

Received: 05 Nov. 2016

First Revision: 27 Dec. 2017

Accepted: 18 Feb. 2017

Key Words:

Mitochondrial disorder, MELAS Syndrome,

Middle age

\begin{abstract}
A B S T R A C T
Introduction: Mitochondrial encephalopathy, lactic acidosis, and stroke-like episodes (MELAS) can involve multiple systems and cause stroke-like episodes and status epilepticus.

Case Presentation: A 48-year-old female with history of early fatigability, migraine-type headaches, and bilateral sensory-neural hearing loss presented 3 episodes of serial seizures. On admission she was affected by Wernicke aphasia and, then, right hemiparesis. Investigations showed elevated arterial lactate and ragged red fibers on muscle biopsy.

Conclusion: Though more commonly diagnosed during childhood, some cases of adult-onset MELAS syndrome are reported. This syndrome should be considered in patients with stroke-like events in adults without cerebrovascular risk factors and difficult-to-treat seizures.
\end{abstract}

\section{Introduction}

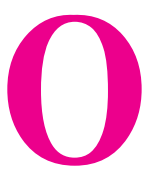

ne of the metabolic mitochondrial disorders is the syndrome of mitochondrial encephalopathy, lactic acidosis, and stroke-like episodes (MELAS), which represents with multisystem involvement (Yoshida et al., 2013) MELAS is the most common mitochondrial myopathy, yet rare with a prevalence of 0.18 per 100000 (Yatsuga et al., 2012). The most common clinical feature of MELAS syndrome is the stroke-like episodes (Kim et al., 1996); however, other features including muscle weakness, headache, vomiting, short stature, dementia, seizure, cardiomegaly, deafness, and endocrinopathies complete the classic clinical scenario (Chae et al., 2004).

The strokes occur in mitochondrial disorders, including MELAS syndrome, tend not to have a vascular distribution, but rather happen in the occipital lobe (Cohen, 2013). These stroke-like episodes may show a variety of neurological symptoms such as migrainous headaches, homonymous hemianopsia, an altered mental status, and seizures (Pavlakis, Phillips, DiMauro, De Vivo, Rowland, 1984). Seizures may occur either as series or even status epilepticus (Yoshida et al., 2013).

The inheritance of MELAS syndrome is in the maternal line and the disease usually manifests from 2 to

* Corresponding Author:

Mostafa Almasi, MD

Address: Department of Neurology, Firoozgar Hospital, Iran University of Medical Sciences, Tehran, Iran.

Tel:+46 (739) 302627

E-mail: a_mostafa108@yahoo.com 
10 years old (Pavlakis et al., 1984). Nevertheless, there are few cases with disease-onset during late adulthood up to the age of 62 (Hsu et al., 2012; Gilchrist, Sikirica, Stopa, \& Shanske, 1996; Corr, Gaughan, Moroney, \& Looby, 2014). The current report was a rare case with MELAS syndrome with symptoms represented at the age of above 40 years.

\section{Case Presentation}

A 48-year-old female admitted to the hospital complaining of 3 times generalized tonic-clonic seizures. The seizures occurred at sleep 2 days before admission and decreased level of consciousness lasted until admission. She was taking sodium valproate since two months ago, when her 1st seizures begun. Her attacks appeared as a series of seizures and the patient had decreased level of consciousness for 4 days following seizures. In her past medical history, she had no developmental problems; however, she had a history of bilateral sensory-neural hearing loss since the age of 35 (Figure 1), also migrainetype headaches and early fatigability in recent years. There was no family history of neurologic diseases. During the 1st physical examination, she obeyed only 1-step orders, had fluent speech with neither fever nor neck rigidity nor optic disc swelling. She had gaze preference to left side, but no weakness or Babinski sign was detected.

Laboratory evaluations demonstrated mild anemia (hemoglobin $11.6 \mathrm{~g} / \mathrm{dL}$ ), elevated erythrocyte sedimentation rate $(43 \mathrm{~mm})$, and mild respiratory alkalosis on arterial blood gas analysis. Serum electrolytes, calcium, liver function tests, and creatinine phosphokinase were all normal. Analysis of the cerebrospinal fluids (CSF) was also normal. Initial brain Computed Tomography (CT) scan showed no significant lesion.

At the second day of admission, the patient developed right hemiparesis with muscle force grade 4 of 5 , and Wernicke aphasia. New brain CT scan showed mass effect on the left hemisphere. Diffusion-weighted brain Magnetic Resonance Imaging (MRI) showed large t2-hyperintense lesion in the left parietooccipital lobes with falcine, subfalcine, and right tentorial herniations (Figure 2). Due to suspicion to cerebral vein thrombosis, brain MR-venography was performed, which was normal (Figure 2). Mannitol (20\%, $300 \mathrm{~mL}$ bullous and 150 $\mathrm{mL}$ every 4 hours for 24 hours) and dexamethasone (24 $\mathrm{mg}$ daily in divided doses) were prescribed.

Additional laboratory tests indicated elevated arterial lactate $(24 \mathrm{mg} / \mathrm{dL}$, normal range: 4.5 to $14.5 \mathrm{mg}$ / dL), normal thyroid function tests, antinuclear antibody, anti-cardiolipin antibody, antiphospholipid antibody, rheumatoid factor, and anti-neutrophil cytoplasmic antibody. No cardiac abnormality was observed on electrocardiogram, transthoracic echocardiography, and saline-infusion echocardiography. Results of transcranial ultrasonography and carotid Doppler were normal. Brain conventional angiography showed no arterial occlusion. Electroencephalography (EEG) showed diffuse slow activity with left side sharp waves.

Biopsy specimen was obtained from left deltoid muscle to rule out mitochondrial disorders. Fresh muscle sample was frozen in isopentane cooled in liquid nitrogen. It showed slight fiber-size variation with few internalized nuclei, and some fibers with slight cytoplasmic basophilic discoloration (Figure 3-A). Histochemical studies on frozen muscle sample showed multiple ragged red fibers (the modified Gomori trichrome stain, Figure 3-B). Abnormal peripheral proliferation of mitochondria (succinate dehydrogenase stain) (Figure 3-C) was observed in multiple fibers. There was no COX (combined cytochrome $\mathrm{C}$ oxidase and succinic dehydrogenase stain) negative fiber (Figure 3-D). Finally, the diagnosis of mitochondrial myopathy with COX positive ragged red fibers was suggested, which was compatible with clinical diagnosis of MELAS syndrome.

Level of consciousness and aphasia were improved on the $4^{\text {th }}$ and $12^{\text {th }}$ days of admission, respectively. She was discharged on the $22^{\text {nd }}$ day of admission with mild hemi-

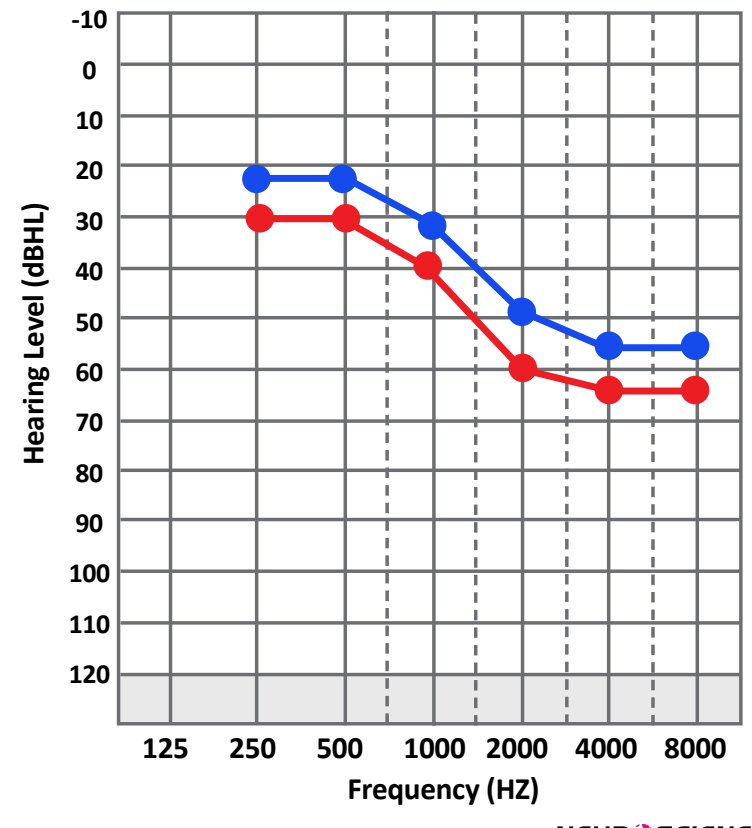

NEUR:SCIENCE

Figure 1. Pure tone audiogram, sensorineural hearing loss (blue line: Air conduction, red line: Bone conduction). 

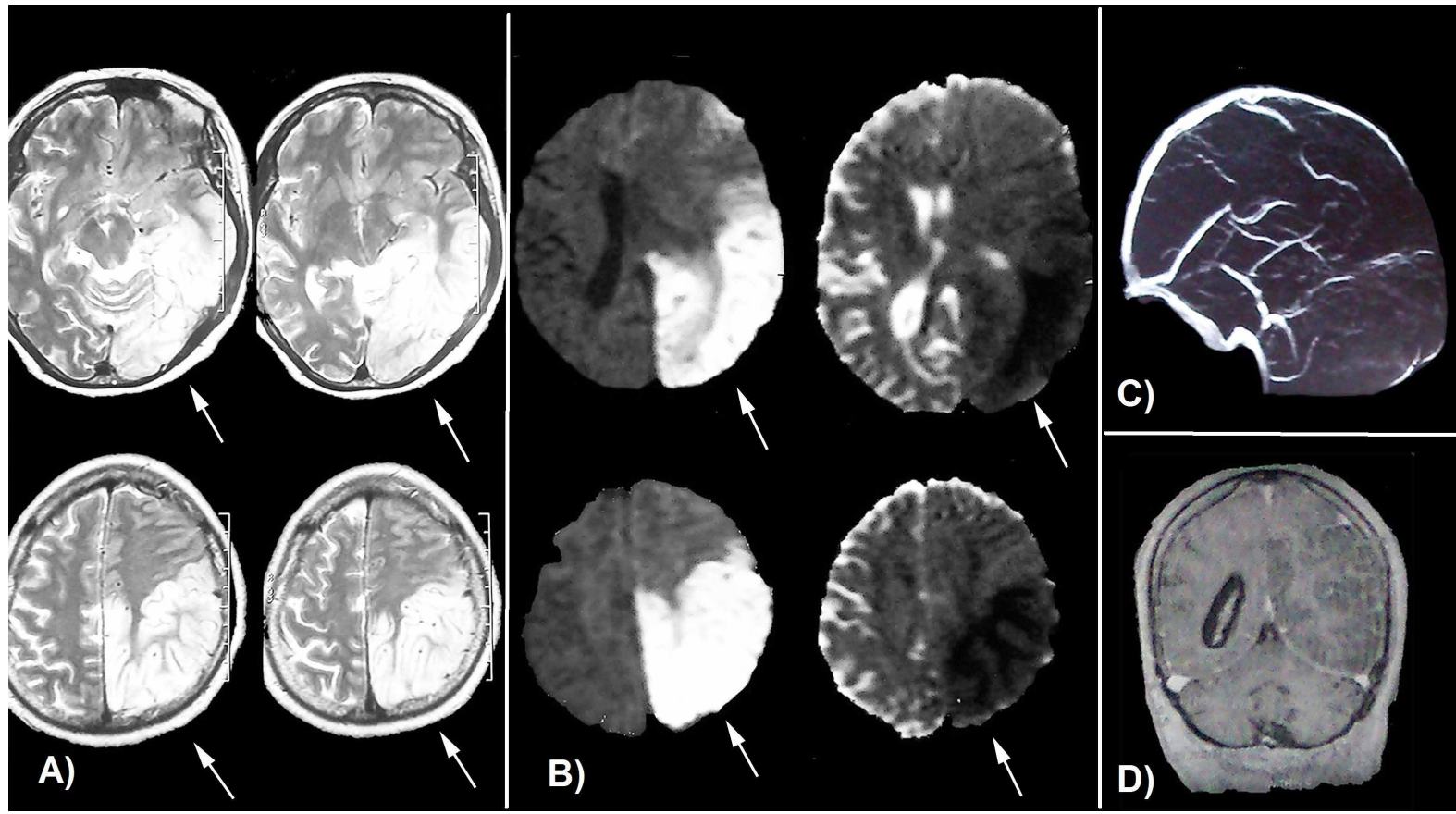

NEUR:SCIENCE

Figure 2. A) Axial t2-weighted brain MRI; hypersignaling in left parietooccipital lobes with mass effect; B) Axial diffusionweighted MRI and ADC show water restriction in left parietooccipital lobes related to acute ischemia; C) Brain MRV shows no abnormality in dural sinuses; D) Coronal t1-weighted brain MRI with contrast injection; hypo-signality in left parietooccipital lobes with mass effect on lateral ventricle without contrast enhancement.

(ADC: Apparent Diffusion Coefficient; MRV: Magnetic Resonance Venography)

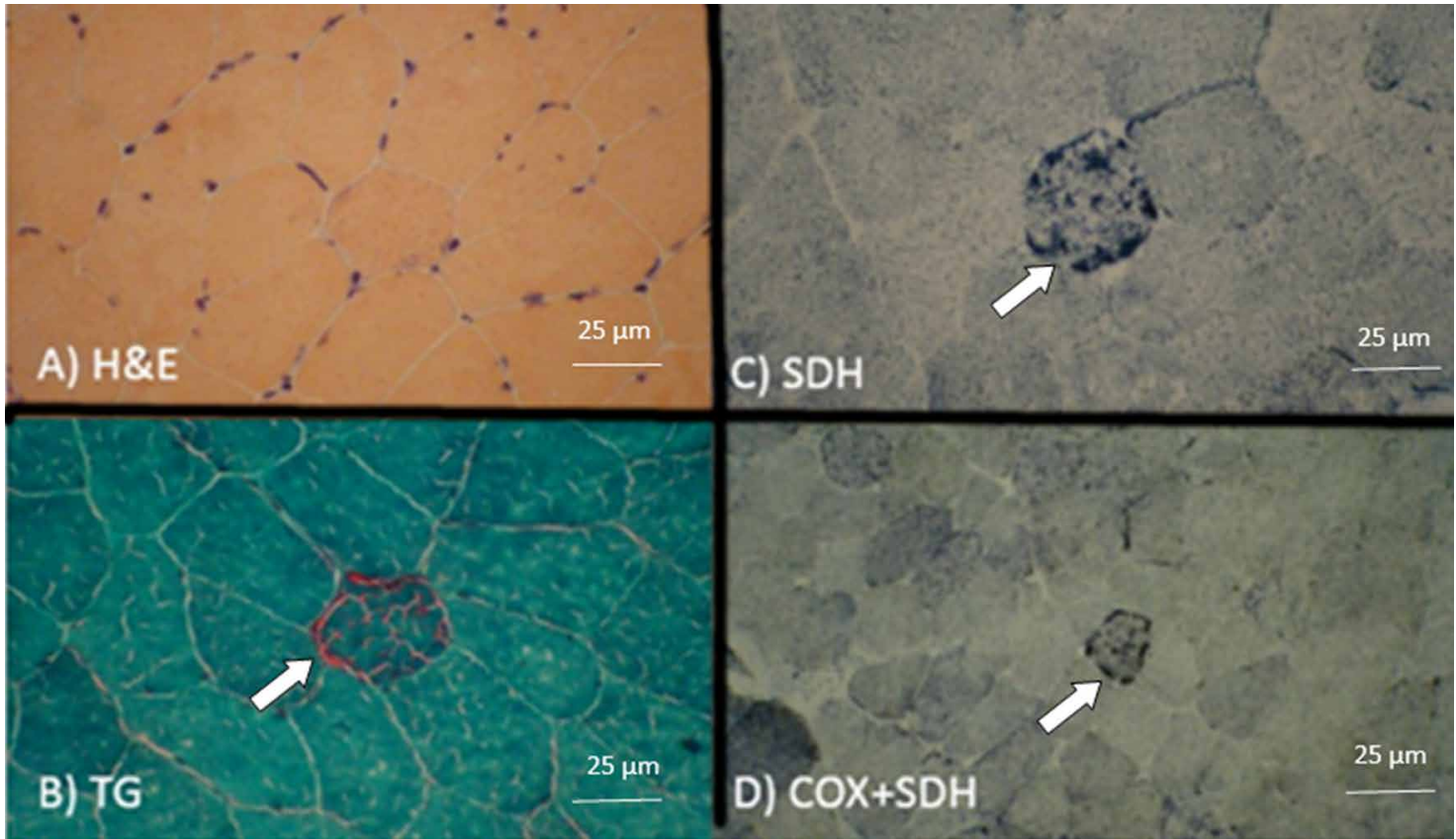

NEUR:SCIENCE

Figure 3. Biopsy specimen from deltoid muscle: A) H \& E staining (400X); fiber size variation with few internalized nuclei and cytoplasmic basophilic discoloration; B) Modified TG staining (400X): ragged red fiber; C) \& D) Abnormal peripheral proliferation of mitochondria in SDH staining and no detection of COX negative fiber in combined COX and SDH staining (400X).

(H \& E: Hematoxylin and Eosin; SDH: Succinate Dehydrogenase; TG : Gomori Trichrome; COX: Cytochrome C Oxidase) 
paresis under treatment with coenzyme-Q 10, L-carnitine, and multivitamins. After 6 months of follow-up, she had neither paresis nor aphasia, and her seizures were controlled by levetiracetam.

\section{Discussion}

The reported patient presented serial seizures followed by hemiparesis and aphasia. Brain imaging showed an ischemic lesion in the left hemisphere predominantly located in occipital lobe. Her aphasia and hemiparesis markedly improved with conservative treatments within 2 weeks. Stroke-like episodes are the most common clinical features of MELAS syndrome (Kim et al., 1996). In addition to hemiparesis, these episodes may present with other neurologic symptoms including migrainous headaches, homonymous hemianopsia, an altered mental status, and seizures (Pavlakis et al., 1984). Seizures, especially status epilepticus or epilepsia partialis continua, which are difficult-to-treat, raise the possibility of mitochondrial disorders (Bindoff \& Engelsen, 2012).

It is more plausible to consider MELAS as a potential diagnosis in patients who have experienced worsened seizure activity under treatment with valproic acid (Hsu et al., 2012). Yoshida et al., reported a patient with 2 episodes of status epilepticus (similar to the patient in the current report) and suggested that a more protracted course of seizures with a persistent altered mental status was concomitant with larger brain lesions (Yoshida et al., 2013). On the other hand, stroke-like episodes as the most common clinical features, and the corresponding changes in brain imaging are very helpful to guide clinicians to correctly diagnose MELAS syndrome (Pauli, Zarzycki, Krzysztalowski, \& Walecka, 2013).

Another important diagnostic procedure in mitochondrial disorders is muscle biopsy. Microscopic changes, such as description of the "ragged-red fibers", which are observed in muscle specimens following staining with modified Gomori trichome, are often associated with mitochondrial myopathies (Cohen, 2013). These disabled mitochondria accumulate in the muscles and manifest as described.

Although the basic pathophysiology of stroke-like episodes is unknown, alterations in nitric oxide homeostasis and over-reduction/oxidative stress are recommended as the 2 leading mechanisms (lizuka \& Sakai, 2010). In clinical practice, partial effectiveness of immediate and long-term therapy with L-arginine and Lcitrulline to prevent subsequent strokes (Jobgen, Fried, $\mathrm{Fu}$, Meininger, $\mathrm{Wu}, 2006$ ) suggests that the role of nitric oxide metabolism is very important.
Most of the cases with MELAS syndrome manifest their 1st features when they are 2 to 10 years old (Pavlakis et al., 1984). In recent years, there were some rare case reports presenting MELAS syndrome during adulthood (Hsu et al., 2012; Gilchrist et al., 1996; Corr et al., 2014). Vrettou et al. (2013) reported a 56-year-old patient presenting with status epilepticus and hypo-density in the left temporal lobe in brain CT scan. Also, a Taiwanese patient was reported with stroke-like episodes at the age of 62 (Li et al., 2007). Mitochondrial disorders progress at different rates (Cohen, 2013) and it could be possible that some factors such as nutrition, physical and behavioral activities, contact with oxidative stressors, and antioxidants may cause delayed evolution of their symptoms.

In conclusion, MELAS syndrome is one of the mitochondrial disorders with a variety of symptoms and should be considered in adult patients with stroke-like events without cerebrovascular risk factors and difficultto-treat seizures.

\section{Acknowledgements}

This research did not receive any specific grant from funding agencies in the public, commercial, or not-forprofit sectors

\section{Conflict of Interest}

The authors declared no conflicts of interest.

\section{References}

Bindoff, L. A., \& Engelsen, B. A. (2012). Mitochondrial diseases and epilepsy. Epilepsia, 53, 92-97. doi: 10.1111/j.15281167.2012.03618.x

Chae, J. H., Hwang, H., Lim, B. C., Cheong, H. I., Hwang, Y. S., \& Kim, K. J. (2004). Clinical features of A3243G mitochondrial tRNA mutation. Brain and Development, 26(7), 459-462. doi: 10.1016/j.braindev.2004.01.002

Cohen, B. H. (2013). Neuromuscular and systemic presentations in adults: diagnoses beyond MERRF and MELAS. Neurotherapeutics, 10(2), 227-242. doi: 10.1007/s13311-013-0188-3

Corr, A., Gaughan, M., Moroney, J., \& Looby, S. (2014). MELAS, an important consideration in the adult population presenting with unusual and recurrent stroke-like episodes. BMJ Case Reports, 2014. doi: 10.1136/bcr-2013-201640

Gilchrist, J. M., Sikirica, M., Stopa, E., \& Shanske, S. (1996) Adult-onset melas: Evidence for involvement of neurons as well as cerebral vasculature in strokelike episodes. Stroke, 27(8), 1420-1423. doi: 10.1161/01.str.27.8.1420 
Hsu, Y. C., Yang, F. C., Perng, C. L., Tso, A. C., Wong, L. J. C., \& Hsu, C. H. (2012). Adult-onset of mitochondrial myopathy, encephalopathy, lactic acidosis, and stroke-like episodes (MELAS) syndrome presenting as acute meningoencephalitis: A case report. The Journal of Emergency Medicine, 43(3), 163-166. doi: 10.1016/j.jemermed.2009.10.021

lizuka, T., \& Sakai, F. (2010). Pathophysiology of stroke-like episodes in MELAS: neuron-astrocyte uncoupling in neuronal hyperexcitability. Future Neurology, 5(1), 61-83. doi: 10.2217/ fnl.09.71

Jobgen, W. S., Fried, S. K., Fu, W. J., Meininger, C. J., \& Wu, G. (2006). Regulatory role for the arginine-nitric oxide pathway in metabolism of energy substrates. Journal of Nutritional Biochemistry, 17(9), 571-588. doi: 10.1016/j.jnutbio.2005.12.001

Kim, I. O., Kim, J. H., Kim, W. S., Hwang, Y. S., Yeon, K. M., \& Han, M. C. (1996). Mitochondrial myopathy-encephalopathylactic acidosis-and strokelike episodes (MELAS) syndrome: $\mathrm{CT}$ and MR findings in seven children. American Journal of Roentgenology, 166(3), 641-645. doi: 10.2214/ajr.166.3.8623642

Li, J. Y., Hsieh, R. H., Peng, N. J., Lai, P. H., Lee, C. F., Lo, Y. K., et al. (2007). A follow-up study in a Taiwanese family with mitochondrial myopathy, encephalopathy, lactic acidosis and stroke-like epi-sodes syndrome. Journal of the Formosan Medical Association, 106(7), 528-536. doi: 10.1016/s0929-6646(07)600035

Pauli, W., Zarzycki, A., Krzysztalowski, A., \& Walecka, A. (2013). $\mathrm{CT}$ and MRI imaging of the brain in MELAS syndrome. Polish Journal of Radiology, 78(3), 61-65. doi: 10.12659/PJR.884010

Pavlakis, S. G., Phillips, P. C., DiMauro, S., De Vivo, D. C., \& Rowland, L. P. (1984). Mitochondrial myopathy, encephalopathy, lactic acidosis, and strokelike episodes: A distinctive clinical syndrome. Annals of Neurology, 16(4), 481-488. doi: 10.1002/ana.410160409

Vrettou, C. S., Zervakis, D., Priovolos, A., Koskina, S., Tsamouri, M., \& Routsi, C. (2013). MELAS syndrome diagnosed in ICU in a 56-year-old patient presenting with status epilepticus. Intensive Care Medicine, 39(6), 1148-1149. doi: 10.1007/s00134013-2884-1

Yatsuga, S., Povalko, N., Nishioka, J., Katayama, K., Kakimoto, N., Matsuishi, T., et al. (2012). MELAS: A nationwide prospective cohort study of 96 patients in Japan. Biochimica et Biophysica Acta (BBA)-General Subjects, 1820(5), 619-624. doi: 10.1016/j. bbagen.2011.03.015

Yoshida, T., Ouchi, A., Miura, D., Shimoji, K., Kinjo, K., Sueyoshi, T., et al. (2013). MELAS and reversible vasoconstriction of the major cerebral arteries. Internal Medicine, 52(12), 1389 92. doi: 10.2169 /internalmedicine.52.0188 


\section{Authors' Guidelines}

Basic and Clinical Neuroscience is devoted to rapid publication of original research works which are of great interest to the broad community of neuroscientists. Manuscripts in all areas of neuroscience including molecular, cellular, developmental, systems, behavioral and cognitive, and related fields will be considered for publication.

Submission: Submission of a manuscript to Basic and Clinical Neuroscience must constitute of original research not previously published, and that it is not being considered for publication elsewhere. Manuscripts submitted under multiple authors are reviewed on the assumption that all listed authors have agreed on the submission and that a copy of the final manuscript has been approved by all authors. Manuscripts are first reviewed by the section editors to ensure their appropriateness relevant to the framework of the journal. The manuscript would be peer-reviewed by related specialists. If accepted, the article shall not be published elsewhere, without the consent of the Editors and Publisher.

\section{Declaration of Copyright in the journal website}

Manuscripts of length up to a maximum of 8000 words will be considered for publication. Articles should be written in English, and double spaced with wide margins. The layout and style should adhere strictly to the instructions given and in particular, the reference style of Basic and Clinical Neuroscience. The author(s) must declare that all experiments on human subjects were conducted with the adequate understanding and written consent of the subjects. When experimental animals are used, the methods section must clearly indicate that adequate measures were taken to minimize pain or discomfort. All animal experiments must be approved by local animal care and use committees.

\section{Ethical Committee}

Submission items include Cover Letter, Reviewer Suggestions (at least 3), Manuscript including Title Page, $\mathrm{Ab}$ stract, Main text (Original Paper: Introduction, Materials and Methods, Results, and Discussion), Acknowledgements, References and Tables/Figure Legends, Tables and Figures. Text files (double-spaced) of the manuscript should be supplied in Microsoft Word format.
Authors should submit their manuscript through online submission process of $\mathrm{BCN}$ website: http://bcn.tums. ac.ir

And for further information please contact us with the BCN mail Box: bcnj@tums.ac.ir

Title Page: This page should contain the following items: (i) complete title (preferably no chemical formulas or arbitrary abbreviations); (ii) full names of all authors; (iii) complete affiliations of all authors; (iv) the number of text pages of the whole manuscript (including Figures and tables) and the number of Figures and tables; (v) running title; (vi) the name and complete address of the corresponding author (as well as telephone number, facsimile number and E-mail address, and if available URL address) to whom correspondence and proofs should be sent.

Running title: A maximum of 40 characters with spaces should be provided.

Abstract: An Abstract of up to 250 words describing the purpose of the study, the methods, the results, and the conclusion should precede the main text.

Keywords: A maximum of 6 keywords or phrases should be provided; preferably, these should be selected from the body of the text.

Main text: In general, the main text of the manuscript should be organized as follows: Introduction, Materials and Methods, Results, Discussion, Acknowledgements, and References.

References: The authors are responsible for the accuracy of bibliographic information. Citations in the text should conform to the referencing style used by the last version of American Psychological Association (APA) style, http://www.apastyle.org. Examples of reference citations in text:

Single author:

(Adam, 1992) OR Adam (1992) proved that...

Two authors:

(Ringsven \& Morse, 1996) OR In their study, Ringsven and Morse (1996)... 
Three to five authors:

First citation: (Lupton, Brunn, \& Platt, 2000) OR Lupton, Brunn and Platt (2000)...

Subsequent citations: (Johnson et al., 2002)

Six or more authors:

(White et al., 2001) OR White et al., (2001)...

In addition, all references cited in the text should be listed at the end of the manuscript on a separate page in alphabetical order by authors' last names. All items in the list of references should be cited in the text and, conversely, all references cited in the text must be presented in the list. Personal communications and unpublished data including manuscripts submitted, but not yet accepted for publication should not be used as a reference, nonetheless, they may be placed in parentheses in the text.

\section{Reference to a journal publication:}

Senden, T. J., Moock, K. H., Gerald, J. F., Burch, W. M., Bowitt, R. J., \& Ling, C. D., et al. (1997). The physical and chemical nature of technigas. Journal of Nuclear Medicine, 38(10), 1327-1333.

Sanchez, D., \& King-Toler, E. (2007). Addressing disparities consultation and outreach strategies for university settings. Consulting Psychology Journal: Practice and Research, 59(4), 286-295. doi:10.1037/10659293.59.4.286.

\section{Reference to a book:}

Frank, R. H., \& Bernanke, B. (2007). Principles of macro-economics (3rd ed.). Boston: McGraw-Hill/Irwin.

Flower, L., Hayes, J. R., Carey, L., Schriver, K., \& Stratman, J. (1986). Detection, diagnosis, and strategies of revision. In L. Ede (Ed.), The Braddock essays (pp. 191-228). Boston: Bedford St. Martin's.

Illustrations: Illustrations will appear either across a single column $(8 \mathrm{~cm})$ or a whole page $(15 \mathrm{~cm})$. The illustrations should be numbered in Arabic numerals according to the sequence of appearance in the text, where they are referred to as Figure 1, Figure 2, etc. Figure legends should be concise and clear and should not duplicate the body of the text. Each illustration must have a title and an explanatory legend. The title should be part of the legend and not be reproduced on the Figure itself. The legends should be placed on a separate page at the end of the manuscript and begin with the number of the illustration they refer to. All symbols and abbreviations used in the Figure must be explained. Also, please indicate their appropriate locations in the manuscript.

Tables: Tables should be so constructed that they, together with their captions and legends, will be intelligible with minimal reference to the text. Tables of numerical data should each be typed (with double-spacing) on a separate page, numbered in sequence in Arabic numerals (Table 1,2 , etc.) and referred to in the text as Table 1 , Table 2, etc. The title of the table should appear above it. A detailed description of its contents and footnotes should be given below the body of the table. Also, please indicate their appropriate locations in the manuscript.

Conflicts of interest: Authors should disclose, at the time of submission, information on financial conflicts of interest or other interests that may influence the manuscript. Authors should declare sources of funding for the work undertaken.

Proofs: Authors will receive proofs by email. Only printer's errors may be corrected; no change in, or additions to, the edited manuscript will be allowed at this stage. The corrected proofs must be returned within 72 hours after receipt by email. If the Publisher receives no reply, the assumption will be made that there are no errors to correct and the article will be published.

Reprints: A total of 5 reprints of each paper will be provided free of charge to the author(s). 